\title{
WHITE LIGHT DIFFRACTION PHASE MICROSCOPY
}

\author{
K. Sandeep Kumar ${ }^{1}$, Vimal Prabhu Pandiyan², Renu John ${ }^{3}$ \\ ${ }^{I}$ Student of M.Tech., Department of Biomedical Engineering, IIT, Hyderabad, India \\ ${ }^{2}$ Student of Ph.D., Department of Biomedical Engineering, IIT, Hyderabad, India \\ ${ }^{3}$ Associate Professor, Department of Biomedical Engineering, IIT, Hyderabad, India
}

\begin{abstract}
Quantitative phase imaging (QPI) techniques are very advantageous compared to the qualitative phase imaging techniques like bright field microscopy, phase contrast microscopy, differential interference contrast microscopy due to measurement of phase information quantitatively. In QPI techniques, there is no need of exogenous contrast agents to stain or tag the specimen. White light diffraction phase microscopy $(w D P M)$ is one of the QPI techniques which is used to quantify the phase information from the samples. The phase information retrieved is dependent on the local refractive index and thickness of the biological cells. So, by measuring the phase one can find the refractive index and/or thickness of the specimen in nanometer scales. In this technique, the acquisition speed is only limited by the sensing device due to its single shot feature, it is better compared to other quantitative phase imaging techniques as its common path geometry provides better temporal phase sensitivity by cancelling out the most mechanisms responsible for noise and with the use of white light source the images are speckle-free. So we achieve better spatial phase sensitivity compared to other techniques. This method has attractive applications in biomedicine. We have developed wDPM set up for extracting phase information from the live yeast cells and sperm cells. The phase is reconstructed from the recorded interferograms by using a fast phase reconstruction algorithm. This algorithm avoids the unwrapping step which is needed in conventional phase reconstruction by Hilbert's transform method. We have calculated the thickness of the yeast cells and sperm cells and demonstrate a video for visualizing the dynamics of yeast cells.
\end{abstract}

Keywords: Phase Imaging, Coherence imaging, Microscopy, 3-D imaging

\section{INTRODUCTION}

Various biological samples are transparent in nature when illuminated by visible light and they behave as phase objects. There is no significant absorption or scattering of light. In phase objects, the variations in thickness and refractive index cause the phase transformation of the light passing through them. Human eye can perceive only intensity variations but not phase variations. Optical microscopic techniques are widely used for diagnosing and investigating in medicine. The structural view is rendered by transforming the phase information into intensity distribution in phase contrast microscopy and differential interference contrast microscopic techniques [1]. These techniques provide only the qualitative information about phase shift introduced by the specimen and quantitative phase is not feasible. Fluorescence microscopy uses fluorescent dyes for providing contrast between different structures inside the specimen. Live cell imaging cannot be achieved through this technique as staining or tagging results in toxicity leading to the damage of cell, there by prohibiting the live cell imaging for longer periods of time.

\subsection{Quantitative Phase Imaging}

Quantitative phase imaging (QPI) techniques helps in obtaining the phase information quantitatively. QPI combines the ideas of Abbe's interference phenomenon [2], Zernike's image contrast [3, 4] and Gabor's holography [5]. The measured image in QPI is a map of path-length shifts associated with the specimen. The local thickness and refractive index of the internal structures of the sample is quantified in these images. Hilbert phase microscopy (HPM) is an off-axis QPI technique where single interferogram is recorded in the image plane and so the acquisition rates are high as it is limited only by the recording device. HPM is employed for quantifying the dynamics of cell membrane fluctuations at millimeter-nanometer scales and neural activity at millisecond range [6]. Fourier phase microscopy (FPM) is a common path interferometric QPI technique that combines the principles of phase contrast microscopy and phase shifting interferometry where in, scattered and unscattered light from the sample act as object and reference fields respectively [7]. Phase is calculated from the four interferograms recorded by changing the phase of unscattered light in steps of $0, \pi / 2, \pi$ and $3 \pi / 2$ with the help of programmable phase modulator [8]. FPM is used to measure nanoscale fluctuations in the membrane of erythrocytes with time scales from few seconds to hours. DPM is the technique which combines both the HPM and FPM. In this paper we demonstrate wDPM set up for extracting phase information from the live yeast cells and sperm cells. The phase has been reconstructed from the recorded interferograms by employing a fast phase reconstruction algorithm. This algorithm avoids the unwrapping step which is needed in conventional phase reconstruction by Hilbert's transform method. We have calculated the thickness of the yeast cells and sperm cells and recorded a video for visualizing the dynamics of yeast cells. 


\section{DIFFRACTION PHASE MICROSCOPY}

Diffraction phase microscopy (DPM) is a quantitative phase imaging technique that combines the ideas of HPM's singleshot feature with the common path geometry associated with FPM [9]. Due to off-axis geometry, DPM has fast acquisition rates and common path geometry accomplishes high temporal phase sensitivity [10,11]. As laser illumination is employed in DPM, there is an artifact that appears as speckle noise in DPM images. So the spatial phase sensitivity is very low, as a result, the study of subcellular structures is inhibited. By using white light illumination, the speckle noise can be removed there by resulting in high spatial sensitivity. White light DPM helps in recording single shot interferograms with high spatial as well as temporal phase sensitivity. We have developed the optical set up of white light diffraction phase microscopy. This is basically, an add-on module to the commercially available Axio Observer Z1, Zeiss microscope. Figure 1 explains the optical set up of the experiment. A halogen lamp acts as source of white light illumination. The condenser aperture is set to minimum possible value i.e., $\mathrm{NA}=0.09$ so as to make the entire light in the field of view spatially coherent. During the whole process of experiment we operate the microscope in bright filed mode with 40x (0.75 NA) objective [9]. The image is formed at the image plane IP1 outside the inverted microscope. We have used a 4f system (lenses L1, L2) to replicate the microscopic image at plane IP2. We employed reflective spatial light modulator (SLM) that serves the purpose of diffraction phase grating $\mathrm{G}$, in the image plane IP2 with $9 \mu \mathrm{m}$ grating period such that it is smaller than the diffraction-limited spot of the microscopic imaging system at the grating plane [12]. This diffraction grating renders multiple diffraction orders that has full spatial information about the specimen [9]. The two diffraction orders, zeroth and first, are filtered out at the Fourier plane of lens L3. The zeroth order is spatially lowpass filtered through the pin hole such that only DC component of zeroth order is allowed and the diameter of the pin hole is typically maintained at $200 \mu \mathrm{m}$. The entire frequency content of first diffraction order is allowed to pass through the first order mask which is a rectangular opening, typically of the size $5 \times 2 \mathrm{~mm}^{2}$, in the aluminum foil. The lens L3-L4 forms a highly stable Mach-Zehnder interferometer [12]. The first order gives imaging field and zeroth order gives reference field. Then after the two diffraction orders are inverse Fourier transformed by passing through the lens L4, they interfere and generate a spatially modulated interference image which is recorded using a CCD camera [9].

\section{PHASE RECONSTRUCTION}

The quantitative phase image of the sample is retrieved from the recorded interferogram using a fast phase reconstruction algorithm that bypasses the need for unwrapping step that is necessary in conventional Hilbert's transform based reconstruction method [13]. As the unwrapping step is not needed, the phase reconstruction can be done rapidly. At the beginning of the experiment we capture the background interferogram only once, as the phase of the background doesn't change for particular specimen-holder [13].

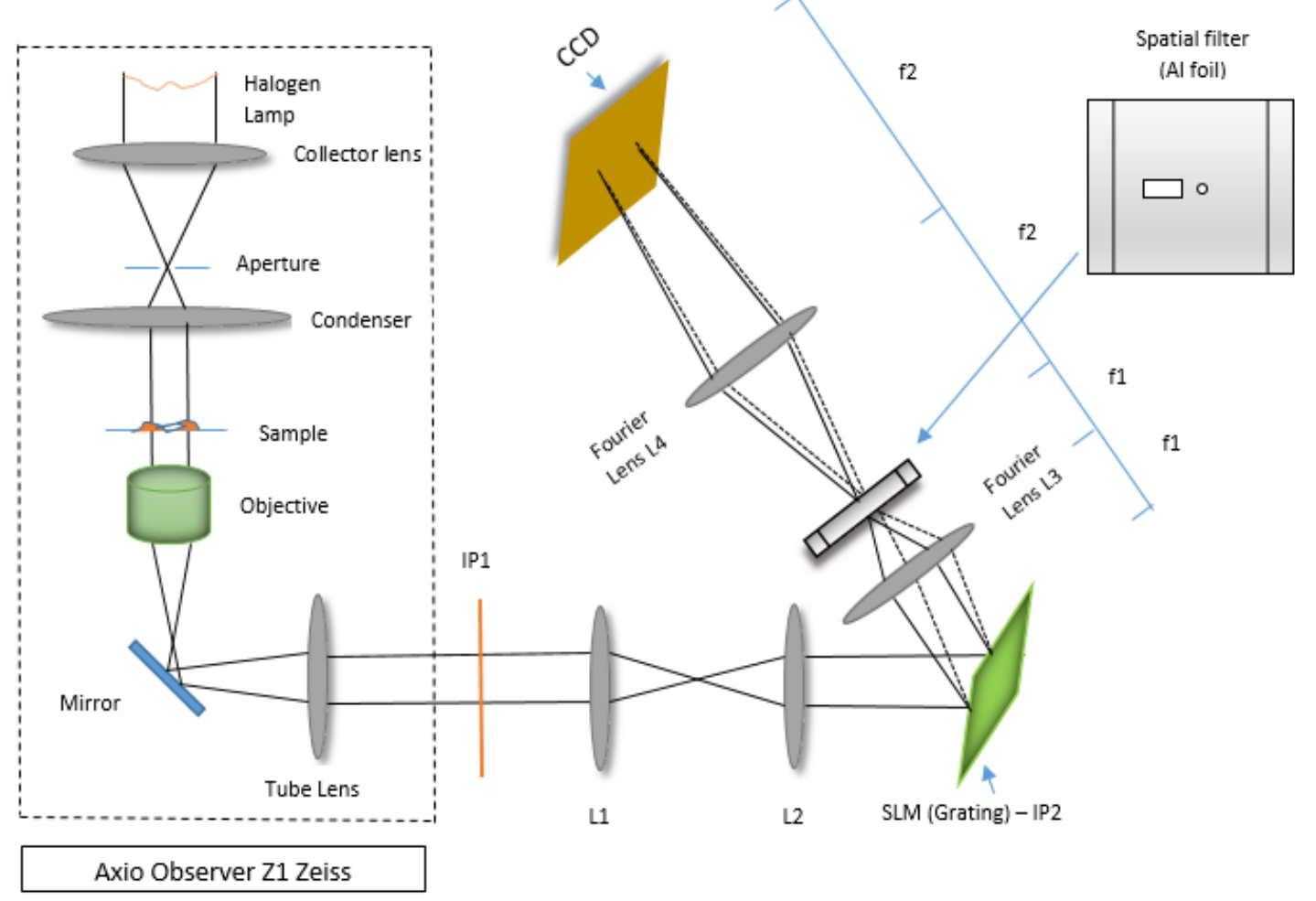

Fig -1: Experimental Setup. 
This background information is used for all the subsequent images. The intensity distribution of the interferogram at the CCD by taking into consideration the background and noise is:

$$
\begin{array}{r}
\mathrm{I}(\mathrm{x}, \mathrm{y})=\left|\mathrm{U}_{\mathrm{i}}(\mathrm{x}, \mathrm{y})\right|^{2}+\left|\mathrm{U}_{\mathrm{r}}\right|^{2}+2\left|\mathrm{U}_{\mathrm{r}}\right| \cdot\left|\mathrm{U}_{\mathrm{i}}(\mathrm{x}, \mathrm{y})\right| \cdot \cos [k \mathrm{x}+ \\
\emptyset \mathrm{x}, \mathrm{y}+\varphi \mathrm{bgx}, \mathrm{y}+\varphi n \mathrm{x}, \mathrm{y}
\end{array}
$$

where $U_{i}(x, y)$ is the imaging field, $U_{r}$ is the reference field, $k$ is the period of grating, $\varnothing(\mathrm{x}, \mathrm{y})$ is the phase delay introduced by the sample, $\varphi_{\mathrm{bg}}(\mathrm{x}, \mathrm{y})$ is the phase shift introduced by the background and $\varphi_{\mathrm{n}}(\mathrm{x}, \mathrm{y})$ is the noise [13]. We Fourier transform the Eq. (1), shift first order to the center and perform the inverse Fourier transform [13], then the resultant term is

$$
2\left|\mathrm{U}_{\mathrm{r}}\right| \cdot\left|\mathrm{U}_{\mathrm{i}}(\mathrm{x}, \mathrm{y})\right| \cdot \exp \left[i\left(\Delta \emptyset(\mathrm{x}, \mathrm{y})+\varphi_{\mathrm{bg}}(\mathrm{x}, \mathrm{y})+\varphi_{\mathrm{n}}(\mathrm{x}, \mathrm{y})\right)\right]
$$

When there is no phase object in the field of view, Eq. (1) becomes [13]:

$$
\begin{aligned}
& \mathrm{I}(\mathrm{x}, \mathrm{y})= \\
& \left|\mathrm{U}_{\mathrm{i}}(\mathrm{x}, \mathrm{y})\right|^{2}+\left|\mathrm{U}_{\mathrm{r}}\right|^{2}+ \\
& \quad 2\left|\mathrm{U}_{\mathrm{r}}\right| \cdot\left|\mathrm{U}_{\mathrm{i}}(\mathrm{x}, \mathrm{y})\right| \cdot \cos \left[k \mathrm{x}+\varphi_{\mathrm{bg}}(\mathrm{x}, \mathrm{y})+\right. \\
& \varphi n \mathrm{x}, \mathrm{y}
\end{aligned}
$$

By following the same process done to Eq. (1), the resultant term for Eq. (3) is [13]:

$$
2\left|\mathrm{U}_{\mathrm{r}}\right| \cdot\left|\mathrm{U}_{\mathrm{i}}(\mathrm{x}, \mathrm{y})\right| \cdot \exp \left[i\left(\varphi_{\mathrm{bg}}(\mathrm{x}, \mathrm{y})+\varphi_{\mathrm{n}}(\mathrm{x}, \mathrm{y})\right)\right]
$$

Dividing Eq. (2) by Eq. (4), we get the term

$$
\exp [i \Delta(\varnothing(x, y)]
$$

Hence, by taking the angle of Eq. (5), we retrieve the phase map associated with the sample.

\section{RESULTS}

We have recorded the phase map of yeast cells and sperm cells. Yeast cells were grown in YPD broth (Yeast extract $1 \%$, peptone $2 \%$ and dextrose $2 \%$ ) till log phase [14]. We have extracted yeast cells for imaging using WDPM technique while they are in log phase. We have used the fast phase reconstruction algorithm for retrieving phase information. We employed UI- 1482LE-M camera that has pixel size of $2.2 \mu \mathrm{m}$, optical sensor diagonal $d$ of $7.04 \mathrm{~mm}$ and resolution $2560 \times 1920$. Figure 2 shows the recorded hologram of yeast cells and Figure 3 shows reconstructed phase map using the fast phase reconstruction algorithm. We have recorded the DPM images for sperm cells after fixation and resuspended in PBS and reconstructed phase by using the fast phase reconstruction algorithm. Figure 4 shows the recorded hologram for sperm cells. Figure 5 shows the reconstructed phase map. By using Eq. (6), we calculated the thickness of yeast cells and sperm cells.

$$
h(x, y)=\frac{\Delta \emptyset(x, y) \lambda}{2 \pi \Delta n}
$$

$h(x, y)$ is the local thickness of the specimen, $\Delta \phi(x, y)$ is the local phase shift introduced by the specimen, $\lambda$ is the mean wavelength of the illumination source which is $532 \mathrm{~nm}$ and $\Delta n$ is the refractive index difference between the specimen and medium. Refractive index of yeast cell is 1.51 and refractive index of YPD medium surrounding yeast is 1.339. So the thickness of the yeast cell is calculated to be $1.3 \mu \mathrm{m}$. Refractive index of sperm cell is 1.53 and of PBS medium is 1.334 . We calculated the thickness of sperm cell to be $773 \mathrm{~nm}$.

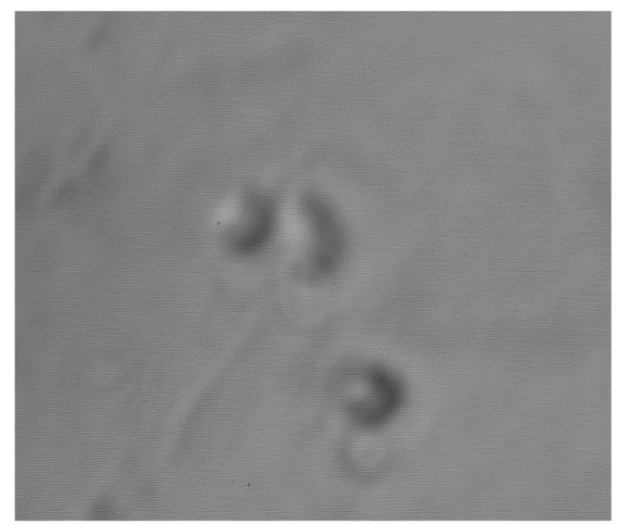

Fig -2: Hologram of yeast cells

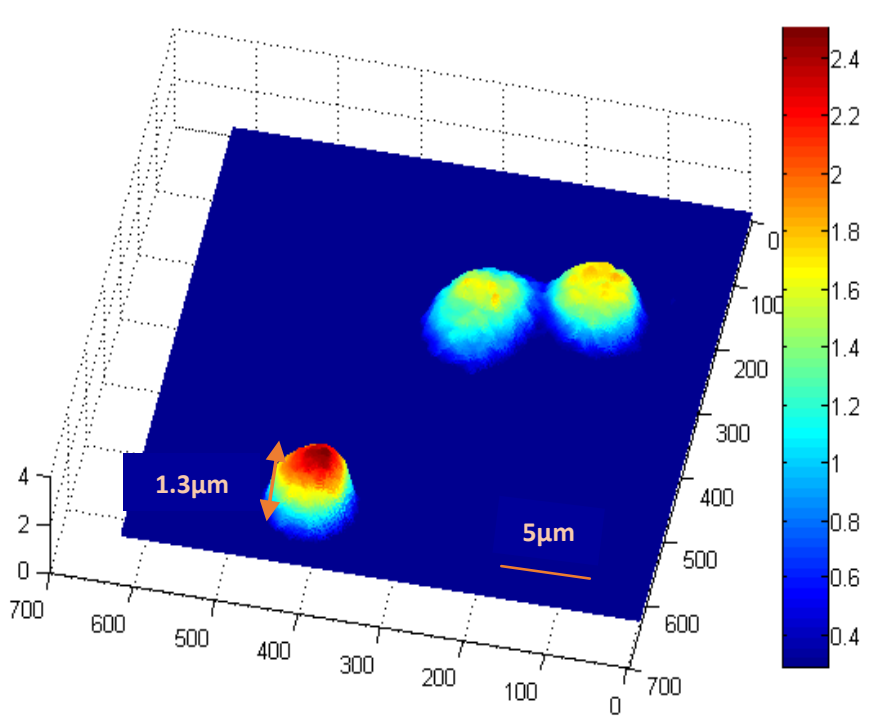

Fig -3: Reconstructed 3-d Phase 


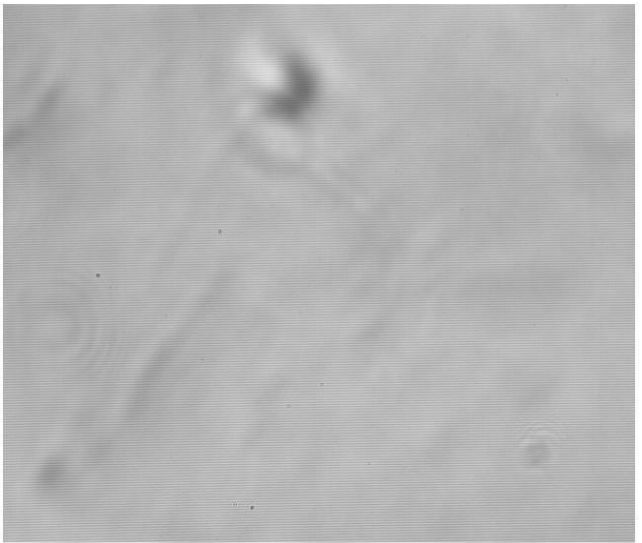

Fig -4: Hologram of Sperm cells

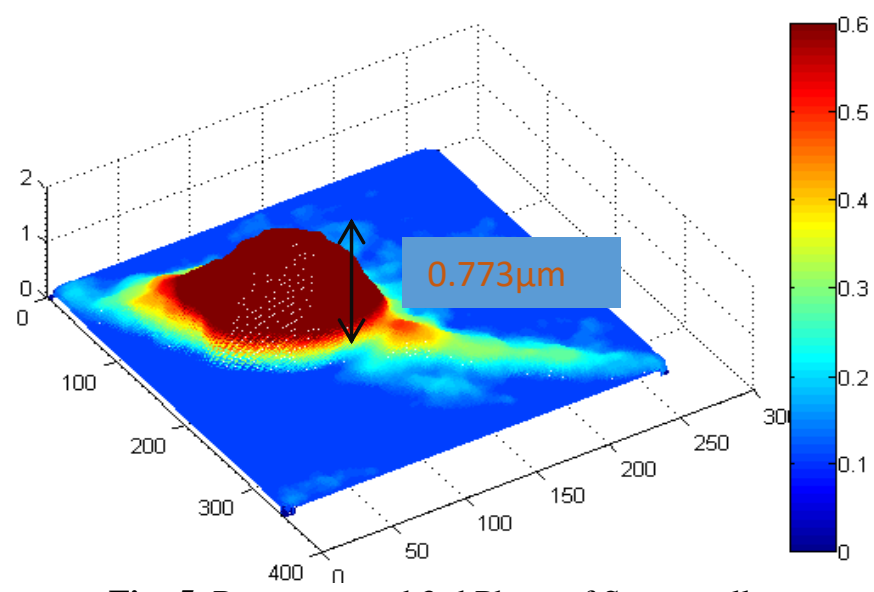

Fig -5: Reconstructed 3-d Phase of Sperm cells

\section{CONCLUSION}

We have demonstrated the phase imaging of yeast cells and sperm cells using whit light diffraction phase microscopy. We have calculated the thickness of the yeast and sperm cells from the phase maps which are in hundreds of nanometer and are able to detect the structural fluctuations to nanometer scales. The dynamics of the yeast cells video will be demonstrated in the conference. Also in this paper, we have implemented fast phase reconstruction algorithm in white light diffraction phase microscopy for $3 \mathrm{D}$ volumetric reconstruction of yeast cells and sperm cells. This algorithm bypasses the need for unwrapping and increases the speed of reconstruction. Due to off-axis geometry, DPM has require single shot and common path geometry accomplishes high temporal phase sensitivity. White light illumination of this technique has the advantage of speckle free imaging. In future this technique can be explored for tomographic imaging.

\section{REFRENCES}

[1] Preza, Chrysanthe, Sharon King, Nicoleta Dragomir, and Carol Cogswell. "Phase Imaging Microscopy: Beyond Dark-Field, Phase Contrast, and Differential Interference Contrast Microscopy", Handbook of Biomedical Optics, 2011.

[2] Abbe, E. (1873). Beiträge zur Theorie des Mikroskops und der mikroskopischen
Wahrnehmung. Archiv für mikroskopische Anatomie, 9, 431 .

[3] Zernike, F. (1942b). Phase contrast, a new method for the microscopic observation of transparent objects, Part 1. Physica, 9, 686-698.

[4] Zernike, F. (1942a). Phase contrast, a new method for the microscopic observation of transparent objects, Part 2. Physica, 9, 974-986.

[5] Gabor, D. (1948). A new microscopic principle. Nature, 161, 777.

[6] Ikeda, T., et al., Hilbert phase microscopy for investigating fast dynamics in transparent systems. Optics letters, 2005. 30(10): p. 1165-1167.

[7] Bhaduri, Basanta, Krishnarao Tangella, and Gabriel Popescu. "Fourier phase microscopy with white light." Biomedical optics express 4.8 (2013): 14341441.

[8] G. Popescu, L. P. Deflores, J. C. Vaughan, K. Badizadegan, H. Iwai, R. R. Dasari, and M. S. Feld, "Fourier phase microscopy for investigation of biological structures and dynamics," Opt. Lett. 29(21), 2503-2505 (2004).

[9] Bhaduri, Basanta, et al. "Diffraction phase microscopy with white light." Optics letters 37.6 (2012): 1094-1096.

[10] G. Popescu, T. Ikeda, R. R. Dasari, and M. S. Feld, Opt. Lett. 31, 775 (2006).

[11] Y. K. Park, G. Popescu, K. Badizadegan, R. R. Dasari, and M. S. Feld, Opt. Express 14, 8263 (2006).

[12] Bhaduri, Basanta, et al. "Diffraction phase microscopy: principles and applications in materials and life sciences." Advances in Optics and Photonics6.1 (2014): 57-119.

[13] Pham, Hoa V., et al. "Fast phase reconstruction in white light diffraction phase microscopy." Applied optics 52.1 (2013): A97-A101.

[14] Ezeronye, O. U., and P. O. Okerentugba. "Optimum conditions for yeast protoplast release and regeneration in Saccharomyces cerevisiae and Candida tropicalis using gut enzymes of the giant African snail Achatina achatina."Letters in applied microbiology 32.3 (2001): 190-193. 